\title{
Structural Properties of the Unobservable Subspace
}

\author{
Juan Ignacio Mulero Martínez ${ }^{1}$ and Alfonso Baños ${ }^{2}$ \\ ${ }^{1}$ Departamento de Ingeniería de Sistemas y Automática, Universidad Politécnica de Cartagena, Campus Muralla del Mar, \\ 30203 Cartagena, Spain \\ ${ }^{2}$ Departamento de Informática y Sistemas, Facultad de Informática, Universidad de Murcia, Campus de Espinardo, \\ 30071 Murcia, Spain
}

Correspondence should be addressed to Juan Ignacio Mulero Martínez; juan.mulero@upct.es

Received 4 February 2015; Accepted 24 May 2015

Academic Editor: Bin Jiang

Copyright ( 2015 J. I. Mulero Martínez and A. Baños. This is an open access article distributed under the Creative Commons Attribution License, which permits unrestricted use, distribution, and reproduction in any medium, provided the original work is properly cited.

\begin{abstract}
The structural properties of the unobservable subspace are explored. In particular the canonical decomposition of the unobservable subspace as a direct sum of cyclic subspaces as well as the conditions for this subspace to be spectral for the system matrix is studied. These properties are applied to simple input-simple output (SISO) feedback systems by connecting the spectral decomposition of the unobservable subspace to the total cancellation of unobservable modes in the compensator with multiple transmission zeros in the plant.
\end{abstract}

\section{Introduction}

In control theory, a dynamical system $\Sigma$ is a processing element that transforms an input $u$ into an output $y$ both depending on time. When restricted to linear-invariant systems, a realization of $\Sigma$ is usually defined in the statespace by a quadruplet of matrices $\Sigma=(A, B, C, D)$ and a vector of internal states $x$. This quadruplet describes a system of differential equations of the type $\dot{x}=A x+B u, y=$ $C x+D u$. In many occasions it is interesting to analyze the capability of inferring internal states by the knowledge of the outputs; this is called the observability of a system and was firstly introduced by American Hungarian scientist Rudolf E. Kalman, [1]. The set of unobservable states that can not be determined from the outputs has structure of $A$-invariant subspace, $\mathcal{N}$.

The intersection of the unobservable subspace $\mathcal{N}$ with another subspace $W$ is common in control theory. For example, in the Kalman decomposition it is possible to determine the $A$-invariant controllable-unobservable subspace by choosing $W$ as the controllable subspace. Another example is found in the stabilizing solutions of the Riccati equation where $W$ is a stable invariant subspace [2]. In all those cases $W$ is $A$-invariant which implies that $\mathcal{N} \cap W$ is also
$A$-invariant; furthermore, it is usual to require a trivially $A$-invariant intersection, $\mathcal{N} \cap W=\{0\}$. When $W$ is not an $A$-invariant subspace or even not a subspace, something can be stated about the invariance of $\mathcal{N} \cap W$. For instance, the uncontrollable set $W_{\bar{c}}$ is not a subspace but we can build the largest subspace contained in $W_{\bar{c}}$, which is not necessarily $A$-invariant. If $\mathcal{N}$ admits a spectral decomposition, $\mathcal{N} \cap W$ turns out to be a controlled-invariant subspace since $A(\mathcal{N} \cap$ $W) \subseteq \mathcal{N} \cap W \subseteq \mathcal{N} \cap W+\operatorname{span}(B)$, where $\operatorname{span}(B)$ is the subspace generated by the columns of $B$ (for more details of conditioned invariant subspaces the reader is referred to $[3,4])$. Furthermore, if $\mathcal{N}$ has a spectral decomposition every subspace $W \subseteq \mathbb{R}^{n}$ is a conditioned invariant subspace under $A$ with respect to $\mathcal{N}$; that is, $A(\mathcal{N} \cap W) \subseteq \mathcal{N} \cap W \subseteq$ $W$. In the pole placement by output injection it is usual to take an arbitrary subspace $W$ which is conditioned invariant; that is, for any matrix $G,(A+G C) W \subseteq W$. However this condition can be removed when $\mathcal{N}$ is spectral. Thus, for an arbitrary matrix $G, A+\left.G C\right|_{\mathscr{N \cap W}}=\left.A\right|_{\mathcal{N} \cap W}$ and $\mathcal{N} \cap W$ is $(A+G C)$-invariant (also this means that $\mathcal{N} \cap W$ is conditioned invariant).

Recently it was shown that the unobservable subspace plays a central role in the well-posedness of a type of systems described by impulsive differential equations called 
reset control systems [5]. In fact the $A$-invariance of the intersection of the unobservable with an arbitrary subspace seems to be key in the study of these systems.

Motivated for the above applications we analyze the canonical structure of the unobservable subspace as a direct sum of cyclic subspaces. Since the observability concept only involves matrices $A$ and $C$, we restrict the problem to systems of the form $\Sigma=(A, B, C)$, (sometimes called strictly proper systems). The main result is developed in Section 2 where the canonical decomposition is derived by resorting to Kalman's decomposition [6]. Also we deal with the necessary and sufficient conditions for the unobservable subspace to be spectral for $A$. Section 3 is devoted to show some interesting properties of the unobservable cyclic subspaces; additionally the necessary and sufficient conditions of $A$ invariance for the intersection of the unobservable subspace with an arbitrary subspace are analyzed. Section 4 deals with the connection between $A$-spectrality of the unobservable subspace and the existence of multiple transmission zeros in feedback systems.

Throughout the paper we will use the following notation. $\mathbb{R}[s]$ describes the ring of polynomials in the variable $s \in$ $\mathbb{C}$ over $\mathbb{R}$. Given $n$ vectors $\left\{u_{1}, \ldots, u_{m}\right\}$, the vector space generated by these vectors is written as $\operatorname{span}\left(u_{1}, \ldots, u_{m}\right)$. The spectrum of a matrix $A$, that is, the set of eigenvalues of $A$, is denoted by $\sigma(A)$. The eigenspace of $A$ with eigenvalue $\lambda$, that is, the set of eigenvectors of $A$ with the same eigenvalue $\lambda$, is indicated with $E_{\lambda}(A)$ and $\mathcal{N}_{\lambda}(A)$ stands for the root subspace of $A$; it contains the vectors from any Jordan chain of $A$ corresponding to $\lambda . m_{\lambda}(A)$ is the geometric multiplicity of $\lambda \in \sigma(A)$; that is, $m_{\lambda}(A)=\operatorname{dim} E_{\lambda}(A)$.

The realization of a system $\Sigma$ is given by a triplet $(A, B, C)$; that is, $\dot{x}=A x+B u, y=C x$, and $\mathscr{O}(\Sigma)$ represents the observability matrix of $\Sigma$; that is, $\mathcal{O}=\sum_{i=1}^{n} e_{i} \otimes C A^{i}$, where “ $\otimes$ " is the Kronecker product, $A \in \mathscr{M}_{n \times n}(\mathbb{R})$, and $e_{i} \in \mathbb{R}^{n}$ is the $i$ th vector of the standard basis with 1 at the $i$ th entry and the remaining entries set to $0 . \mathcal{N}(M)$ are the nullspace of the matrix $M$ and $Z_{\lambda}(u, A)$ is an $A$-cyclic subspace generated by $u$. If $m=\operatorname{dim} Z_{\lambda}(u, A),(A-\lambda I)^{j} u \neq 0$ for $j<m$ and $(A-\lambda I)^{m} u=0$. The set of transmission zeros of $\Sigma$ is denoted by $\mathscr{Z}\{\Sigma\}$.

The set of unobservable modes is $\Lambda_{\bar{o}}=\{\lambda \in \sigma(A): \exists v \in$ $E_{\lambda}$ such that $\left.C v=0\right\}$ and conversely the set of observable modes is $\Lambda_{o}=\sigma(A) \backslash \Lambda_{\bar{o}}$. Associated with these sets we have the subspaces $W_{o}=\bigoplus_{\lambda \in \Lambda_{o}} \mathcal{N}_{\lambda}(A)$ and $W_{\bar{o}}=\bigoplus_{\lambda \in \Lambda_{\bar{o}}} \mathcal{N}_{\lambda}(A)$.

\section{Canonical Decomposition of the Unobservable Subspace}

Given a strictly proper feedback system $\Sigma=(A, B, C)$ we study the decomposition of the unobservable subspace as a direct sum of cyclic subspaces. In particular we are interested in determining necessary and sufficient conditions for this subspace to be $A$-spectral, that is, when $\mathcal{N}(\mathcal{O})$ is a direct sum of the root spaces $\mathcal{N}_{\lambda}(A)$ where $\lambda \in \sigma(A)$.

In the literature it is shown that $\mathcal{N}(\mathcal{O})$ is $A$-invariant and $\mathbb{R}^{n}=\bigoplus_{\lambda \in \sigma(A)} \mathcal{N}_{\lambda}$; from this it is straightforward to write $\mathscr{N}(\mathcal{O})=\bigoplus_{\lambda \in \sigma(A)}\left(\mathcal{N}(\mathcal{O}) \cap \mathcal{N}_{\lambda}(A)\right)$. If $E_{\lambda}(A)$ denotes the eigenspace for an unobservable mode $\lambda \in \sigma(A)$, it is clear that $E_{\lambda}(A) \cap \mathcal{N}(\mathcal{O}) \neq(0)$. Since $E_{\lambda}(A) \subseteq \mathcal{N}_{\lambda}(A)$ we draw $\mathcal{N}_{\lambda} \cap \mathcal{N}(\mathcal{O}) \neq(0)$. However, what is not so clear is the inclusion $\mathcal{N}_{\lambda} \subseteq \mathcal{N}(\mathcal{O})$ for unobservable modes $\lambda \in \sigma(A)$. In general this result is not true for matrices $A$ without control structure as shown in the example below.

Example 1. Let $\Sigma=(A, C)$ be a linear system with matrices

$$
\begin{aligned}
& A=\left(\begin{array}{lll}
1 & 1 & 1 \\
0 & 1 & 1 \\
0 & 0 & 1
\end{array}\right), \\
& C=\left(\begin{array}{lll}
0 & 1 & 1
\end{array}\right) .
\end{aligned}
$$

In this case $\sigma(A)=\{\lambda=1\}$ and $E_{\lambda}(A)=\left\{e_{1}\right\}$ where $e_{1} \in \mathbb{R}^{3}$ is a vector of the standard basis. From the Popov-BelevitchHautus test (PBH test, [3]) it is easy to check that $C e_{1}=0$ so $\Lambda_{\bar{o}}=\{\lambda\}$. The generalized eigenvectors are $v_{2}=e_{1}+e_{2}$ and $v_{3}=e_{3}$, which are not in the unobservable subspace. Henceforth $\mathcal{N}_{\lambda} \nsubseteq \mathscr{N}(\mathcal{O})$.

Root spaces $\mathcal{N}_{\lambda}(A)$ are decomposed in direct sum of cyclic subspaces so it is worth analyzing the conditions for an $A$-cyclic subspace to be unobservable. As expected this depends on the unobservability of its generator.

Lemma 2. A necessary and sufficient condition for an A-cyclic subspace $Z_{\lambda}(u, A)$ to be unobservable $\left(Z_{\lambda}(u, A) \subseteq \mathcal{N}(\mathcal{O})\right)$ is that $u \in \mathcal{N}(\mathcal{O})$.

Proof. Consider the following.

Sufficiency. Assume that $\operatorname{dim} Z_{\lambda}(u, A)=k$. Let $\mathscr{B}=\{u, A u$, $\left.\ldots, A^{k-1} u\right\}$ be a basis of $Z_{\lambda}(u, A)$. Since $u \in \mathcal{N}(\mathcal{O}), C A^{j} u=0$ for $j=0,1, \ldots, n$. For $j \geq k, A^{j} u$ depends on the vectors in $\mathscr{B}$ which results into $A^{i} u \in \mathcal{N}(\mathcal{O})$ for $i=0,1, \ldots, k-1$.

Necessity. If $Z_{\lambda}(u, A) \subseteq \mathscr{N}(\mathcal{O})$, in particular $u \in \mathcal{N}(\mathcal{O})$.

The following theorem is central in our development and reveals that the unobservable subspace is actually a direct sum of $A$-cyclic subspaces.

Theorem 3. $\mathscr{N}(\mathcal{O})$ can be decomposed into a direct sum of $A$ cyclic subspaces.

Proof. $\mathcal{N}(\mathcal{O})$ is $A$-invariant. From Kalman's decomposition there exists an adapted basis such that $A$ is similar to a matrix $\widetilde{A}=\left(\begin{array}{cc}\widetilde{A}_{o} & 0 \\ \widetilde{A}_{21} & \widetilde{A}_{\bar{o}}\end{array}\right)$ via a matrix $P$; that is, $A=P \widetilde{A} P^{-1}$. We assume that $\operatorname{dim} \mathscr{N}(\mathcal{O})=m$ and adopt the notation $\widetilde{u}=\left(0^{T}, u^{T}\right)^{T}$ for vectors $u \in \mathbb{R}^{m}$ embedded into $\mathbb{R}^{n}$. Let $Z_{\lambda}\left(u, \widetilde{A}_{\bar{o}}\right)$ be an $\widetilde{A}_{\bar{o}}$-cyclic subspace generated by $u$ (Krylov subspace); that is, $Z_{\lambda}\left(u, \widetilde{A}_{\bar{o}}\right)=\operatorname{span}\left(u, \widetilde{A}_{\bar{o}} u, \ldots, \widetilde{A}_{\bar{o}}^{k-1} u\right)$ where $k=$ $\operatorname{dim} Z_{\lambda}\left(u, \widetilde{A}_{\bar{o}}\right) \leq m$. Because $\widetilde{A}^{j}$ is also a lower triangular matrix $\left(\begin{array}{cc}\widetilde{A}_{o}^{j} & 0 \\ * & \widetilde{A}_{\bar{o}}^{j}\end{array}\right)$ we can embed $Z_{\lambda}\left(u, \widetilde{A}_{\bar{o}}\right)$ into $\mathbb{R}^{n}$ through an isomorphism $Z_{\lambda}\left(u, \widetilde{A}_{\bar{o}}\right) \cong Z_{\lambda}(\widetilde{u}, \widetilde{A})$. Generators behave 
well with respect to similarity [7]; that is, $P Z_{\lambda}(\widetilde{u}, \widetilde{A})$ is an $A$-cyclic subspace with generator $P \widetilde{u}$. As a result of this $P Z_{\lambda}(\widetilde{u}, \widetilde{A})=Z_{\lambda}(P \widetilde{\mathcal{u}}, A)$ and $\operatorname{dim} Z_{\lambda}\left(u, \widetilde{A}_{\bar{o}}\right)=\operatorname{dim} Z_{\lambda}(P \widetilde{\mathcal{u}}, A)$. Finally, it is obvious that $P \widetilde{u} \in \mathscr{N}(\mathcal{O})$. In virtue of Lemma 2, $Z_{\lambda}(P \widetilde{u}, A) \subseteq \mathscr{N}(\mathcal{O})$. Thus, $\mathcal{N}(\mathcal{O})$ is the direct sum of cyclic subspaces $P Z_{\lambda}(\widetilde{u}, \widetilde{A})$ with $Z_{\lambda}(\widetilde{u}, \widetilde{A})$ isomorphic to an $\widetilde{A}_{\bar{o}^{-}}$ cyclic subspace $Z_{\lambda}\left(u, \widetilde{A}_{\bar{o}}\right)$.

To illustrate the application of Theorem 3 we provide the following example.

Example 4. Let $\Sigma=(A, C)$ be a linear system with matrices

$$
\begin{aligned}
& A=\left(\begin{array}{cccc}
-2 & -1 & 1 & 2 \\
1 & 0 & -1 & -1 \\
1 & 1 & -1 & 0 \\
0 & 0 & 0 & -1
\end{array}\right), \\
& C=\left(\begin{array}{llll}
1 & 1 & 0 & 0
\end{array}\right) .
\end{aligned}
$$

Now we derive Kalman's decomposition of $\Sigma$ via a similarity transformation $A=P \widetilde{A} P^{-1}$ :

$$
\begin{aligned}
& P=\left(\begin{array}{cccc}
0 & 0 & 1 & 1 \\
1 & 0 & -1 & -1 \\
0 & 0 & 1 & -1 \\
0 & 1 & 0 & 0
\end{array}\right), \\
& \widetilde{A}=\left(\begin{array}{cccc}
-1 & 1 & 0 & 0 \\
0 & -1 & 0 & 0 \\
0 & 1 & -\frac{1}{2} & -\frac{1}{2} \\
-1 & 1 & \frac{1}{2} & -\frac{3}{2}
\end{array}\right) .
\end{aligned}
$$

The unobservable matrix $\widetilde{A}_{\bar{o}}=\left(\begin{array}{cc}-1 / 2 & -1 / 2 \\ 1 / 2 & -3 / 2\end{array}\right)$ has a generator $u=\left(\begin{array}{c}0 \\ -2\end{array}\right)$ and a cyclic subspace $Z_{-1}\left(\widetilde{A}_{\bar{o}}, u\right)$ :

$$
Z_{-1}\left(\widetilde{A}_{\bar{o}}, u\right)=\operatorname{span}\left(u, \widetilde{A}_{\bar{o}} u\right)=\operatorname{span}\left(\left(\begin{array}{c}
0 \\
-2
\end{array}\right),\left(\begin{array}{l}
1 \\
3
\end{array}\right)\right) .
$$

Note that to check that $Z_{-1}\left(\widetilde{A}_{\bar{o}}, u\right)$ is cyclic it is sufficient to observe that $\left(\widetilde{A}_{\bar{o}}-\lambda I\right)^{2} u=0$ and $\left(\widetilde{A}_{\bar{o}}-\lambda I\right) u \neq 0$.

We embed $Z_{-1}\left(\widetilde{A}_{\bar{o}}, u\right)$ into $\mathbb{R}^{4}$ by defining $\widetilde{u}=$ $(0,0,0,-2)^{T}$. Again we can check that $\widetilde{u}$ is a generator of the cyclic subspace $Z_{-1}(\widetilde{A}, \widetilde{u})=\operatorname{span}((0,0,0,-2),(0,0,1,3))$ by the conditions $\left(\widetilde{A}_{\bar{o}}-\lambda I\right)^{2} \widetilde{u}=0$ and $\left(\widetilde{A}_{\bar{o}}-\lambda I\right)^{2} \widetilde{u} \neq$ 0 . As expected $Z_{-1}(\widetilde{A}, \widetilde{u})$ is isomorphic to $Z_{-1}\left(\widetilde{A}_{\bar{o}}, u\right)$ since the embedding is injective and $\operatorname{dim} Z_{-1}(\widetilde{A}, \widetilde{u})=Z_{-1}\left(\widetilde{A}_{\bar{o}}, u\right)$. Finally, $P \widetilde{u}=(-2,2,2,0)$ is the generator of a cyclic subspace $Z_{-1}(A, P \widetilde{u})=\operatorname{span}(P \widetilde{u}, A P \widetilde{u}):$

$$
\begin{aligned}
(A-\lambda I)^{2} P \widetilde{u} & =P(\widetilde{A}-\lambda I)^{2} P^{-1}(P \widetilde{u})=P(\widetilde{A}-\lambda I)^{2} \widetilde{u} \\
& =0, \\
(A-\lambda I) P \widetilde{u} & =P(\widetilde{A}-\lambda I) \widetilde{u} \neq 0 .
\end{aligned}
$$

In the last identity note that $P$ is an isomorphism and $(\widetilde{A}-$ $\lambda I) \widetilde{u} \neq 0$ which results in $P(\widetilde{A}-\lambda I) \widetilde{u} \neq 0$. It is also easy to see that $P \widetilde{u} \in \mathcal{N}(\mathcal{O})$ since $C P \widetilde{u}=0$.

Corollary 5. If $\mathbb{R}^{n}$ admits a canonical decomposition in direct sum,

$$
\mathbb{R}^{n}=\bigoplus_{\lambda \in \sigma(A)} \bigoplus_{k=1}^{m_{\lambda}(A)} Z_{\lambda}\left(u_{k}, A\right)
$$

then the unobservable subspace $\mathcal{N}(\mathcal{O})$ can be decomposed in direct sum:

$$
\mathcal{N}(\mathcal{O})=\bigoplus_{\lambda \in \Lambda_{\bar{o}}} \bigoplus_{l=1}^{m_{\lambda}(A)} \mathcal{N}\left(\mathcal{O}\left(C,\left.A\right|_{Z_{\lambda}\left(u_{l}, A\right)}\right)\right)
$$

where $\mathcal{N}\left(\mathcal{O}\left(C,\left.A\right|_{Z_{\lambda}\left(u_{1}, A\right)}\right)\right)=\cap_{k=0}^{n-1} \mathcal{N}\left(C,\left.A^{k}\right|_{Z_{\lambda}\left(u_{l}, A\right)}\right)=Z\left(v_{l}, A\right)$ with $v_{l}$ being a generalized eigenvector of $\left.A\right|_{Z_{\lambda}\left(u_{l}, A\right)}$.

It is worth emphasizing that the above direct decomposition of $\mathscr{N}(\mathcal{O})$ in cyclic subspaces may not include all the cyclic subspaces associated with Jordan blocks (from now on and for the sake of brevity $A$-cyclic subspaces from the Jordan decomposition of $\mathbb{R}^{n}$ will be referred to as Jordan $A$ cyclic subspaces) $J_{\lambda}(A)$ where $\lambda \in \Lambda_{\bar{o}}$. This occurs when the Jordan cyclic subspace $Z_{\lambda}(u, A)$ has an eigenvector $v$ such that $C v \neq 0$ or more generally when $Z_{\lambda}(A, u) \cap \mathscr{N}(\mathcal{O})^{\perp} \neq$ $\varnothing$. Furthermore the cyclic subspaces of the decomposition in Theorem 3 may not coincide with those of the Jordan canonical decomposition. What is clear is that the cyclic subspaces in the decomposition of $\mathcal{N}(\mathcal{O})$ are included in Jordan A-cyclic subspaces as stated in the following corollary.

In the following proposition we state necessary and sufficient conditions for $\mathcal{N}(\mathcal{O})$ to be $A$-spectral.

Proposition 6 (spectral subspace). Given a system $\Sigma=$ $(A, B, C)$ its unobservable subspace $\mathcal{N}(\mathcal{O})$ is a spectral subspace for $A$ if and only if every $\mathcal{N}_{\lambda} \subseteq \mathcal{N}(\mathcal{O})$ for all $\lambda \in \Lambda_{\bar{o}}$.

Proof. Assume that $\mathcal{N}(\mathcal{O})$ is spectral for $A$; that is, $\mathcal{N}(\mathcal{O})$ is a direct sum of root spaces $\mathscr{N}_{\lambda}$ for $A$ where $\lambda \in \sigma(A)$. This decomposition means that if $\mathcal{N}_{\lambda} \cap \mathcal{N}(\mathcal{O}) \neq(0)$ then $\mathcal{N}_{\lambda} \subseteq$ $\mathcal{N}(\mathcal{O})$. Since $E_{\lambda}\left(\mathcal{N}_{\lambda}\right) \cap \mathcal{N}(\mathcal{O}) \neq(0)$ for all $\lambda \in \Lambda_{\bar{o}}$, we conclude that $\mathcal{N}_{\lambda} \subseteq \mathcal{N}(\mathcal{O})$ for all $\lambda \in \Lambda_{\bar{o}}$.

Now we prove the converse. Assume that $\mathcal{N}_{\lambda} \subseteq \mathscr{N}(\mathcal{O})$ for all $\lambda \in \Lambda_{\bar{o}}$ and define $W_{\bar{o}} \triangleq \bigoplus_{\lambda \in \Lambda_{\bar{o}}} \mathcal{N}_{\lambda}, W_{o} \triangleq \bigoplus_{\lambda \in \Lambda_{o}} \mathcal{N}_{\lambda}=$ $W_{\bar{o}}^{\perp}$. We prove the inclusion $W_{\bar{o}} \subseteq \mathcal{N}(\mathcal{O})$. Because root spaces are maximal invariant subspaces it is clear that $\mathbb{R}^{n}$ admits a decomposition in direct sum $\mathbb{R}^{n}=W_{\bar{o}} \oplus W_{o}$. We define the $A$ invariant subspace $W=W_{o} \cap \mathcal{N}(\mathcal{O})$. By reductio ad absurdum assume that $W \neq(0)$. The linear transformation $A$ restricted to $W,\left.A\right|_{W}$, has an eigenvalue $\lambda$ and an eigenvector $w \in W$. In addition we have the chain of inclusions $W \subseteq \mathcal{N}(\mathcal{O}) \subseteq$ $\mathcal{N}(C)$ which means that $C w=0$; that is, $\lambda$ is an unobservable mode of $\left.A\right|_{W_{o}}$ (restriction of $A$ to $W_{o}$ ). However, the modes of $\left.A\right|_{W_{o}}$ are those in $\Lambda_{o}$. This contradiction proceeds from the fact that $W$ was nonempty. Henceforth, $W=(0)$, and $\mathcal{N}(\mathcal{O})$ is spectral for $A$. 


\section{Structural Properties of the Unobservable Subspace}

We begin analyzing the $A$-cyclic subspaces that appear in the canonical decomposition of $\mathcal{N}(\mathcal{O})$, which will be called unobservable subspaces. We point out that every unobservable cyclic subspace lies in one Jordan $A$-cyclic subspace, as the following corollary suggests.

Corollary 7. Let $Z_{\lambda}(v, A)$ be an unobservable $A$-cyclic subspace as in Theorem 3. Then there exists a Jordan A-cyclic subspace $Z_{\lambda}(u, A)$ such that $Z_{\lambda}(v, A) \subseteq Z_{\lambda}(u, A)$.

Proof. The vector $v$ is in a Jordan $A$-cyclic subspace; that is, there exists a generator $u$ such that $v \in Z_{\mu}(u, A)$ for some eigenvalue $\mu \in \sigma(A)$. Since $Z_{\mu}(u, A)$ is $A$-invariant then vectors $A^{k} v$ are in $Z_{\mu}(v, A)$. Therefore $Z_{\lambda}(v, A) \subseteq Z_{\mu}(u, A)$ and $\lambda=\mu$.

Example 8. The matrix $A$ in Example 4 has only an $A$-cyclic subspace $Z_{-1}(v, A)$ with generator $v=(0,1,-1,1) . Z_{-1}(v, A)$ has generalized eigenvectors $v_{1}=v, v_{2}=(0,1,1,0)$, and $v_{3}=(0,0,1,0)$ and eigenvector $v_{4}=(1,-1,0,0)$. As shown above $Z_{-1}(P \tilde{u}, A) \subseteq \mathcal{N}(\mathcal{O})$ with generator $P \widetilde{u}=(-2,2,2,0)^{T}$. It is easy to see that this generator is a linear combination of generalized eigenvectors of $\left.A\right|_{Z_{-1}(v, A)}, P \widetilde{u}=2 v_{3}-2 v_{4}$. Thus $Z_{-1}(P \tilde{u}, A) \subseteq Z_{-1}(v, A)$.

Lemma 2 can also be proved as a consequence of Corollary 7. We only show the sufficiency since the necessity is trivial: $u \in \mathscr{N}(\mathcal{O})$ implies $u \in Z_{\lambda}(v, A) \subseteq \mathscr{N}(\mathcal{O})$ for some unobservable generator $v$. By definition of cyclic subspace, $Z_{\lambda}(u, A)$ is the minimal subspace that contains the generator $u$. Therefore $Z_{\lambda}(u, A) \subseteq Z_{\lambda}(v, A)$, and in virtue of Corollary $7, Z_{\lambda}(v, A) \subseteq Z_{\lambda}(w, A)$ for some generator $w$. From the decomposition of $\mathbb{R}^{n}$ in $A$-cyclic subspaces it is known that $Z_{\lambda}(w, A) \cap Z_{\lambda}(u, A)=(0)$ if $w \notin \operatorname{span}(u)$. However $u \in Z_{\lambda}(v, A) \cap Z_{\lambda}(u, A) \subseteq Z_{\lambda}(w, A) \cap Z_{\lambda}(u, A)$ and then $Z_{\lambda}(w, A) \cap Z_{\lambda}(u, A) \neq(0)$. As a result, $Z_{\lambda}(v, A) \subseteq Z_{\lambda}(w, A)=$ $Z_{\lambda}(u, A)$, and $Z_{\lambda}(u, A)=Z_{\lambda}(v, A)$.

That lemma reveals that a sufficient condition for a Jordan $A$-cyclic subspace $Z_{\lambda}(u, A)$ to match with $Z_{\lambda}(v, A)$ in the decomposition of $\mathcal{N}(\mathcal{O})$ is justly $u \in Z_{\lambda}(v, A)$. This is reasonable since in that case the Jordan $A$-cyclic subspace should be in $\mathcal{N}(\mathcal{O})$.

Not only is every unobservable $A$-cyclic subspace in a Jordan $A$-cyclic subspace as revealed in 2 , but also the former inherits successive generalized eigenvectors from the latter. This means that we can easily build a basis for the unobservable $A$-cyclic subspace by choosing a subchain of generalized eigenvectors, from the Jordan $A$-cyclic subspace, ending up in an eigenvector. This idea is stated in the following lemma.

Lemma 9. If $Z(u, A)=\operatorname{span}\left(u_{1}, u_{2}, \ldots, u_{m}\right) \subseteq Z(v, A)=$ $\operatorname{span}\left(v_{1}, v_{2}, \ldots, v_{r}\right)$ with $r \geq m$ and $u_{k}, v_{k} \neq 0$ are generalized eigenvectors, that is,

$$
\begin{aligned}
& u_{1}=u, \\
& v_{1}=v,
\end{aligned}
$$

$$
\begin{aligned}
& (A-\lambda I) u_{k}=u_{k+1} \text { for } k=1, \ldots, m-1, \\
& (A-\lambda I) v_{k}=v_{k+1} \quad \text { for } k=1, \ldots, r-1, \\
& (A-\lambda I) u_{m}=0, \\
& (A-\lambda I) v_{r}=0,
\end{aligned}
$$

then $Z(u, A)=\operatorname{span}\left(v_{r-m+1}, v_{r-m+2}, \ldots, v_{r}\right)$.

Proof. Firstly let us write the eigenvector $u_{m} \in Z_{\lambda}(v, A)$ as a linear combination of the vectors in the basis of $Z_{\lambda}(v, A)$, that is, $u_{m}=\sum_{k=1}^{r} \alpha_{k}^{(m)} v_{k}$, and multiply it on the left by $(A-\lambda I)$ :

$$
(A-\lambda I) u_{m}=0=\sum_{k=1}^{r-1} \alpha_{k}^{(m)} v_{k+1} .
$$

Since $\left\{v_{2}, \ldots, v_{r}\right\}$ are linearly independent it follows that $\alpha_{k}^{(m)}=0$ for $k=1, \ldots, r-1$. Consequently $u_{m}$ depends linearly on $v_{r}$. and

As for $u_{m-1} \in Z_{\lambda}(A, v)$ we write $u_{m-1}=\sum_{k=1}^{r} \alpha_{k}^{(m-1)} v_{k}$

$$
(A-\lambda I)^{2} u_{m-1}=0=\sum_{k=1}^{r-2} \alpha_{k}^{(m-1)} v_{k+2}
$$

Again $\left\{v_{3}, \ldots, v_{r}\right\}$ are linearly independent so $\alpha_{k}^{(m-1)}=0$ for $k=1, \ldots, r-2$. Thus $u_{m-1}$ depends linearly on $v_{r-1}$ and $v_{r}$.

Proceeding similarly we find that $u_{j}$ depends on $\left\{v_{r-m+j}, \ldots, v_{r}\right\}$ for $j=1, \ldots, m$ and we obtain the following triangular arrangement:

$$
\begin{aligned}
& \left(\begin{array}{c}
u_{1} \\
u_{2} \\
\vdots \\
u_{m-1} \\
u_{m}
\end{array}\right) \\
& \left(\begin{array}{cccccccc}
0 & 0 & \cdots & \alpha_{r-m+1}^{(1)} & \alpha_{r-m+2}^{(1)} & \cdots & \alpha_{r-1}^{(1)} & \alpha_{r}^{(1)} \\
0 & 0 & \cdots & 0 & \alpha_{r-m+2}^{(2)} & \cdots & \alpha_{r-1}^{(2)} & \alpha_{r}^{(2)} \\
\vdots & \vdots & \ddots & \vdots & \vdots & \ddots & \vdots & \vdots \\
0 & 0 & \cdots & 0 & 0 & \cdots & \alpha_{r-1}^{(m-1)} & \alpha_{r}^{(m-1)} \\
0 & 0 & \cdots & 0 & 0 & \cdots & 0 & \alpha_{r}^{(m)}
\end{array}\right)\left(\begin{array}{c}
v_{1} \\
v_{2} \\
\vdots \\
v_{r-1} \\
v_{r}
\end{array}\right) .
\end{aligned}
$$

From this it is evident that, for $j=r-m+1, \ldots, r, v_{j}$ depends linearly on $\left\{u_{m-r+j}, \ldots, u_{m}\right\}$ and then $v_{j} \in Z_{\lambda}(u, A)$. Owing to the fact that vectors $v_{r-m+1}, \ldots, v_{r}$ are linearly independent they constitute a basis for $Z_{\lambda}(u, A)$. Henceforth, $Z_{\lambda}(u, A)=$ $\operatorname{span}\left(v_{r-m+1}, v_{r-m+2}, \ldots, v_{r}\right)$. 
Example 10. In Example 8, $Z_{-1}(P \tilde{u}, A)=\operatorname{span}\left(u_{1}, u_{2}\right) \subseteq$ $Z_{-1}(v, A)=\operatorname{span}\left(v_{1}, v_{2}, v_{3}, v_{4}\right)$, where

$$
\begin{aligned}
& v_{1}=(0,1,-1,1), \\
& v_{2}=(0,1,1,0), \\
& v_{3}=(0,0,1,0), \\
& v_{4}=(1,-1,0,0), \\
& u_{1}=(-2,2,2,0)=P \widetilde{u}=2 v_{3}-2 v_{4}, \\
& u_{2}=(A+I) u_{1}=(2,-2,0,0)=2 v_{4} .
\end{aligned}
$$

Applying back substitution $v_{4}=(1 / 2) u_{2}, v_{3}=(1 / 2) u_{1}+$ $(1 / 2) u_{2}$, then $v_{3}, v_{4} \in Z_{-1}(P \widetilde{u}, A)$ and $Z_{-1}(P \widetilde{u}, A)=$ $\operatorname{span}\left(v_{3}, v_{4}\right)$.

Example 11. Consider the system $\Sigma=(A, C)$ with

$$
\begin{aligned}
& A=\left(\begin{array}{ccccccc}
-1 & 0 & 0 & 0 & 0 & 0 & 0 \\
0 & -1 & 0 & 0 & -1 & 0 & 0 \\
1 & 0 & -1 & 0 & 0 & 0 & 0 \\
0 & 0 & 0 & -1 & 0 & 1 & 0 \\
0 & 0 & 0 & 0 & -2 & 0 & 0 \\
0 & 1 & 0 & 0 & -1 & -1 & 0 \\
0 & 0 & 0 & 0 & 1 & 0 & -2
\end{array}\right), \\
& C=\left(\begin{array}{lllllll}
0 & 1 & 0 & 0 & 0 & 0 & 0
\end{array}\right) \text {. }
\end{aligned}
$$

We have the canonical Jordan decomposition of $R^{7}$ in cyclic subspaces:

$$
\mathbb{R}^{7}=Z_{-2}\left(e_{2}+e_{5}, A\right) \oplus Z_{-1}\left(e_{1}, A\right)+\oplus Z_{-1}\left(e_{2}, A\right) .
$$

From Corollary 5 the unobservable subspace can be decomposed as

$$
\begin{aligned}
& \mathcal{N}(O) \\
& =\mathcal{N}\left(\mathcal{O}\left(C,\left.A\right|_{Z_{-2}\left(e_{2}+e_{5}, A\right)}\right)\right) \\
& \quad \oplus \mathcal{N}\left(\mathcal{O}\left(C,\left.A\right|_{Z_{-1}\left(e_{1}, A\right)}\right)\right) \\
& \quad \oplus \mathcal{N}\left(\mathcal{O}\left(C,\left.A\right|_{Z_{-1}\left(e_{2}, A\right)}\right)\right) \\
& \mathcal{N}\left(\mathcal{O}\left(C,\left.A\right|_{Z_{-2}\left(e_{2}+e_{5}, A\right)}\right)\right)=\operatorname{span}\left(e_{7}\right)=Z_{-2}\left(e_{7}, A\right), \\
& \mathcal{N}\left(\mathcal{O}\left(C,\left.A\right|_{Z_{-1}\left(e_{1}, A\right)}\right)\right)=\operatorname{span}\left(e_{1}, e_{3}\right)=Z_{-1}\left(e_{1}, A\right), \\
& \mathcal{N}\left(\mathcal{O}\left(C,\left.A\right|_{Z_{-1}\left(e_{2}, A\right)}\right)\right)=\operatorname{span}\left(e_{4}, e_{6}\right)=Z_{-1}\left(e_{4}, A\right) .
\end{aligned}
$$

Therefore,

$$
\mathcal{N}(O)=Z_{-2}\left(e_{7}, A\right) \oplus Z_{-1}\left(e_{1}, A\right) \oplus Z_{-1}\left(e_{4}, A\right) .
$$

Note that $e_{7}$ and $e_{1}$ are eigenvectors of the Jordan cyclic subspaces $Z_{-2}\left(e_{2}+e_{5}, A\right)$ and $Z_{-1}\left(e_{1}, A\right)$, respectively, while $e_{4}$ is an eigenvector of $Z_{-1}\left(e_{2}, A\right)$. The last fact is a consequence of Lemma 9 since $Z_{-1}\left(e_{4}, A\right) \subseteq Z_{-1}\left(e_{2}, A\right)$ and $\operatorname{dim} Z_{-1}\left(e_{4}, A\right)=$ 2.
Above in Lemma 2 we have shown that every unobservable $A$-cyclic subspace is determined by the unobservability of its generator. Now we state the converse result for an $A$ cyclic subspace to be in the observable subspace. (It is very obvious that the set of observable states has no structure of subspace. However, we can consider the observable subspace as the orthogonal complement of the unobservable subspace, $\mathscr{N}(\mathcal{O})^{\perp}$.) In this case the observability of the eigenvector of the $A$-cyclic subspace determines the observability of the whole subspace. This is proved in the following lemma.

Lemma 12. If $v \in E_{\lambda}(A) \cap \mathscr{N}(\mathcal{O})^{\perp}$ and $v \in Z_{\lambda}(u, A)$ then $Z_{\lambda}(u, A) \subseteq \mathcal{N}(\mathcal{O})^{\perp}$.

Proof. By reductio ad absurdum, assume that $W=Z_{\lambda}(u, A) \cap$ $\mathcal{N}(\mathcal{O}) \neq(0)$. Then $\lambda \in \sigma\left(\left.A\right|_{W}\right)$ and $E_{\lambda}\left(\left.A\right|_{W}\right) \neq(0)$. We take an arbitrary eigenvector $w \in E_{\lambda}\left(\left.A\right|_{W}\right)$. Since $w \in W, C w=$ 0 , but from Lemma $9 w$ depends linearly on $v$ which means $C v=0$ and thus $v \in \mathcal{N}(\mathcal{O})$. This is a contradiction since we assumed by hypothesis that $v \in \mathcal{N}(\mathcal{O})^{\perp}$.

Note that the converse is not always true; that is, $v \in$ $E_{\lambda}(A) \cap \mathcal{N}(\mathcal{O})$ does not necessarily imply $Z_{\lambda}(u, A) \subseteq \mathcal{N}(\mathcal{O})$. In general $\mathcal{N}(\mathcal{O})$ is not spectral for an arbitrary matrix $A$ as shown in the example below.

Example 13. Let $\Sigma=(A, C)$ be a linear system with matrices

$$
\begin{aligned}
& A=\left(\begin{array}{lll}
1 & 1 & 1 \\
0 & 1 & 1 \\
0 & 0 & 1
\end{array}\right), \\
& C=\left(\begin{array}{lll}
0 & 1 & 1
\end{array}\right) .
\end{aligned}
$$

In this case $\sigma(A)=\{\lambda\}$ and $E_{\lambda}=\left\{e_{1}\right\}$ where $e_{1} \in \mathbb{R}^{3}$ is a vector of the standard basis. From the $\mathrm{PBH}$ test it is easy to check that $C e_{1}=0$ so $\Lambda_{\bar{o}}=\{\lambda\}$. The generalized eigenvectors are $v_{2}=e_{1}+e_{2}$ and $v_{3}=e_{3}$, which are not in the unobservable subspace. Henceforth $\mathcal{N}_{\lambda} \nsubseteq \mathcal{N}(\mathcal{O})$.

Finally we address the problem of the $A$-invariance of the intersection of $\mathcal{N}(\mathcal{O})$ with an arbitrary subspace $W \subseteq \mathbb{R}^{n}$. This problem is interesting in the well-posedness of reset control systems.

Lemma 14. A subspace $W \subseteq Z_{\lambda}(A, u)$ is $A$-invariant if and only if $W$ is an A-cyclic subspace.

Proof. The reader is referred to [8].

Corollary 15. A subspace $W \subseteq \mathbb{R}^{n}$ is A-invariant if and only if $W$ is a direct sum of $A$-cyclic subspaces.

Proof. Assume that $\mathbb{R}^{n}$ admits a decomposition in direct sum of $A$-cyclic subspaces $Z_{\lambda}(u, A)$. It is evident that $W$ is $A$ invariant if and only if $W \cap Z_{\lambda}(u, A)$ is $A$-invariant, and from Lemma 14 this occurs whenever $W \cap Z_{\lambda}(u, A)$ is $A$-cyclic.

Due to the unobservable subspace $\mathcal{N}(\mathcal{O})$ is $A$-invariant and in virtue of Corollary 15 it follows that $\mathcal{N}(\mathcal{O})$ is a direct sum of $A$-cyclic subspaces. 
Corollary 16. Let $W$ be a subspace in $\mathbb{R}^{n} . W \cap \mathscr{N}(\mathcal{O})$ is $A$ invariant if and only if $W$ is a direct sum of cyclic subspaces.

Proof. The A-invariance of $W \cap \mathscr{N}(\mathcal{O})$ is a consequence of Corollary 15.

\section{Geometric Multiplicity of the Zeros in Feedback Systems}

This section is devoted to prove that unobservable modes of unit feedback systems (whose components have minimal realizations) always have geometric multiplicity 1 . The geometric multiplicity of a zero was firstly defined by Owens for minimal realizations, [9], and we extend the definition for any system.

Definition 17 (geometric multiplicity of a zero). Given a SISO system $\Sigma$ with a realization $(A, B, C, D)$ not necessarily minimal, the geometric multiplicity of $z \in \mathscr{Z}\{\Sigma\}$ is defined as the dimension of the nullspace of the pencil matrix $\left(\begin{array}{c}z I-A \\ C\end{array}\right)$.

If $A \in \mathbb{R}^{n \times n}$ it is evident that $\operatorname{rank}\left(\begin{array}{cc}A-z I & B \\ C & 0\end{array}\right) \leq n+1$, and the loss of full rank occurs when there exist a vector $v \in \mathbb{R}^{n}$ and a scalar $u \in \mathbb{R}$ such that

$$
\begin{aligned}
(A-z I) v & =-B u, \\
C v & =-D u .
\end{aligned}
$$

Obviously if $\Sigma$ has a minimal realization (i.e., there does not exist zero/pole cancellations), $A-z I$ is invertible which means that $v$ is uniquely determined by $u \neq 0$ (since $u=0$ implies $v=0$ ) and the geometric multiplicity of $z$ is always 1 . If $\Sigma$ has a nonminimal realization there exist two cases:

(i) $u=0$ : It is evident that $z \in \sigma(A)$ and that $v \in \mathcal{N}(z I-$ $A)$. In virtue of the $\mathrm{PBH}$ test, the condition $C v=0$ indicates that $z$ is an unobservable mode and thus the geometric multiplicity is the geometric dimension of the unobservable mode, $\mu_{\lambda}:=\operatorname{dim} \mathcal{N}(z I-A) \cap \mathcal{N}(C)$.

(ii) $u \neq 0$ : In this case $(z I-A) v \in \operatorname{span}(B)$ and $v \in$ $\mathcal{N}(z I-A) \oplus W$ with $\operatorname{dim} W=1$. If the system $\Sigma$ has a proper realization, that is, $D=0$, it follows that $C v=0$, and thus $v \in \mathscr{N}(C)$, and $v \in \mathcal{N}(C) \cap \mathscr{N}(z I-$ $A) \oplus \mathscr{N}(C) \cap W$. It is known that $\operatorname{dim} \mathscr{N}(C) \cap W$ is either 0 or 1 so that the geometric multiplicity is either $\mu_{\lambda}$ or $\mu_{\lambda}+1$. If the system $\Sigma$ has no proper realization, that is, $D \neq 0, u$ is uniquely determined by $v$ according to the relation $u=-(1 / D) C v$; as a consequence the geometric multiplicity of $z$ is $\operatorname{dim} \mathscr{N}(z I-A)+1$.

Now we focus on unit feedback systems $\Sigma=\left(\Sigma_{r}, \Sigma_{p}\right)=$ $(A, B, C)$ where $\Sigma_{r}=\left(A_{r}, B_{r}, C_{r}\right)$ and $\Sigma_{p}=\left(A_{p}, B_{p}, C_{p}\right)$ are minimal realizations.

Lemma 18. Let $\lambda$ be an unobservable mode of $\Sigma$ and assume that $\Sigma_{r}$ and $\Sigma_{p}$ have minimal realizations; then the geometric multiplicity of the zero $\lambda$ is always 1.
Proof. Resorting to the PBH test,

$$
\begin{aligned}
\left(\begin{array}{cc}
A_{p}-\lambda I & B_{p} C_{r} \\
-B_{r} C_{p} & A_{r}-\lambda I
\end{array}\right)\left(\begin{array}{l}
v_{p} \\
v_{r}
\end{array}\right) & =\left(\begin{array}{l}
0 \\
0
\end{array}\right), \\
\left(\begin{array}{ll}
C_{p} & 0
\end{array}\right)\left(\begin{array}{c}
v_{p} \\
v_{r}
\end{array}\right) & =0 .
\end{aligned}
$$

It follows that $v_{r} \neq 0$ : By reductio ad absurdum if $v_{r}=0$, $B_{r}\left(C_{p} v_{p}\right)=0$. If $v_{p} \neq 0$, from the minimality of the realization for $\Sigma_{r}$ and the $\mathrm{PBH}$ criterion, $C_{r} v_{r} \neq 0$, which is impossible since $B_{r} \neq 0$. In virtue of the minimality of $\Sigma_{p} v_{r}=0 \Rightarrow$ $v_{p}=0$, since $\left(\begin{array}{c}\lambda I-A_{p} \\ C_{p}\end{array}\right) v_{p}=0$ if and only if $v_{p}=0$, then $v_{r}=0$ leads to a contradiction since it was assumed that $0 \neq$ $\left(\begin{array}{c}v_{p} \\ v_{r}\end{array}\right) \in \mathcal{N}(\lambda I-A)$.

For $\lambda$ to be a zero of $\Sigma_{p}=\left(A_{p}, B_{p}, C_{p}\right)$ it is necessary that $\lambda I-A_{p}$ to be invertible and this occurs so long as $\lambda \notin \sigma\left(A_{p}\right)$. Assume that $\lambda \in \sigma\left(A_{p}\right)$; the $\mathrm{PBH}$ criterion implies $C_{p} v_{p} \neq 0$ actually it is verified for all eigenvector in $\mathcal{N}\left(\lambda I-A_{p}\right)$, which is absurd from the minimality of $\Sigma_{p}$; thus $\lambda \in \mathscr{E}\left\{\Sigma_{p}\right\}$. Note that $\lambda$ is effectively a zero of $\Sigma_{p}: C_{p}\left(\lambda I-A_{p}\right)^{-1} B_{p}\left(C_{r} v_{r}\right)=0$ since $v_{r} \neq 0$ and from arguments of minimality along with the $\mathrm{PBH}$ criterion $C_{r} v_{r} \neq$ 0 . Henceforth $C_{p}\left(\lambda I-A_{p}\right)^{-1} B_{p}=0$. Owing tothe fact that $\lambda$ is a zero of $\Sigma_{p}, v_{p}$ is uniquely determined by $v_{r}$ via the transformation $v_{p}=\left(\lambda I-A_{p}\right)^{-1} B_{p} C_{r} v_{r}$.

$C_{p} v_{p}=0$ implies $v_{r} \in \mathscr{N}\left(A_{r}-\lambda I\right)$ and for SISO systems $\operatorname{dim} \mathcal{N}\left(A_{r}-\lambda I\right)=1$. Then there exist a vector $u \in \mathbb{R}^{n_{r}}$ and a scalar $\alpha \in \mathbb{R}$ such that $v_{r}=\alpha u$. As a result $C_{r} v_{r}=\alpha\left(C_{r} u\right)$ and $v_{p}$ linearly depends on $\alpha$ :

$$
\left(\begin{array}{c}
v_{p} \\
v_{r}
\end{array}\right)=\left(\begin{array}{c}
\left(\lambda I-A_{p}\right)^{-1} B_{p} C_{r} u \\
u
\end{array}\right) \alpha .
$$

Henceforth the geometric multiplicity of $\lambda$ as an eigenvalue of $A$ is always 1 , and there is only a Jordan block associated with any $\lambda \in \Lambda_{\bar{o}}(A)$.

\section{Transmission Zeros and $A$-Spectrality of the Unobservable Subspace}

In this section we analyze when the unobservable subspace of a feedback SISO system is spectral for the system matrix. To this purpose consider a SISO unit feedback control system with single input $r(t) \in \mathbb{R}$ and single output $y(t) \in \mathbb{R}$. Assume that the plant and the regulator have minimal realizations $\Sigma_{p}=\left(A_{p}, B_{p}, C_{p}\right)$ and $\Sigma_{r}=\left(A_{r}, B_{r}, C_{r}\right)$, respectively:

$$
\begin{aligned}
& \Sigma_{p}=\left\{\begin{array}{l}
\dot{x}_{p}(t)=A_{p} x_{p}(t)+B_{p} e(t) \\
u(t)=C_{p} x_{p}(t),
\end{array}\right. \\
& \Sigma_{r}=\left\{\begin{array}{l}
\dot{x}_{r}(t)=A_{r} x_{r}(t)+B_{r} u(t) \\
y(t)=C_{r} x_{r}(t),
\end{array}\right.
\end{aligned}
$$

where $x_{p} \in \mathbb{R}^{n_{p}}, x_{r} \in \mathbb{R}^{n_{r}}$ are internal states, $e(t)=r(t)-y(t)$ stands for the error signal, and $u(t) \in \mathbb{R}$ represents the control 
law. The closed-loop system $\Sigma=(A, B, C)$ can be written in the state-space by considering the state $x=\left(x_{p}^{T}, x_{r}^{T}\right)^{T}$ of dimension $n=n_{p}+n_{r}$ :

$$
\begin{aligned}
\left(\begin{array}{c}
\dot{x}_{p} \\
\dot{x}_{r}
\end{array}\right) & =\left(\begin{array}{cc}
A_{p} & B_{p} C_{r} \\
-B_{r} C_{p} & A_{r}
\end{array}\right)\left(\begin{array}{c}
x_{p} \\
x_{r}
\end{array}\right)+\left(\begin{array}{c}
0 \\
B_{r}
\end{array}\right) r, \\
y & =\left(\begin{array}{ll}
C_{p} & 0
\end{array}\right)\left(\begin{array}{c}
x_{p} \\
x_{r}
\end{array}\right) .
\end{aligned}
$$

We begin exploring the connection of an unobservable $A$ cyclic subspace to the transmission zeros of the plant. Let $Z_{\lambda}(v, A)$ be such an $A$-cyclic subspace with generator $v$ and dimension $\operatorname{dim} Z_{\lambda}(v, A)=m$. A basis of eigenvectors of $Z_{\lambda}(v, A)$ is given by $\left\{v^{(1)}, \ldots, v^{(m-1)}, v^{(m)}\right\}$ where $v^{(k)}=(A-$ $\lambda I)^{k-1} v$. It is obvious that $v^{(1)}=v$ and $v^{(m)} \in E_{\lambda}(A)$. In virtue of Lemma 2 , a necessary and sufficient condition for $Z_{\lambda}(v, A)$ to be in $\mathscr{N}(\mathcal{O})$ is that $C_{p} v^{(1)}=0$. As result we proceed by checking the tower of generalized eigenvectors, $(A-\lambda I) v^{(k-1)}=v^{(k)}$, from top to bottom. To this end we need the following technical lemmas on zeros of SISO systems.

Lemma 19. A necessary and sufficient condition for $\lambda \in \sigma(A)$ to be unobservable is that $\lambda \in \mathscr{Z}\left(\Sigma_{p}\right) \cap \sigma\left(A_{r}\right) \neq \varnothing$.

Proof. Consider the following.

Sufficiency. Since $\Sigma_{r}$ has a minimal realization, if $\lambda \in \sigma\left(A_{r}\right)$ then for all $v_{r} \in E_{\lambda}\left(A_{r}\right) C_{r} v_{r} \neq 0$ is satisfied. Also the minimal realization of the plant means that $\lambda \in \mathscr{Z}\left(\Sigma_{p}\right)$ implies $\lambda \notin$ $\sigma\left(A_{p}\right)$, which results in the invertibility of $\left(A_{p}-\lambda I\right)$. We can define $v_{p}=-\left(A_{p}-\lambda I\right)^{-1} B_{p} C_{r} v_{r}$ so $C_{p} v_{p}=-C_{p}\left(A_{p}-\right.$ $\lambda I)^{-1} B_{p}=0$, and $\lambda$ is an unobservable mode of $A$.

Necessity. If $\lambda \in \sigma(A)$ is unobservable, in virtue of the PBH's criterion there exists a vector $v=\left(\begin{array}{c}v_{p} \\ v_{r}\end{array}\right) \in E_{\lambda}(A)$ such that $\bar{C} v=C_{p} v_{p}=0$. This condition signifies that $\lambda \in \sigma\left(A_{r}\right)$.

If $\lambda \notin \mathscr{E}\left(\Sigma_{p}\right)$ and $\lambda \notin \sigma\left(A_{p}\right)$, then $v_{p}=-\left(A_{p}-\right.$ $\lambda I)^{-1} B_{p} C_{r}$, and $C_{p} v_{p}=0$ implies $\lambda \in \mathscr{E}\left(\Sigma_{p}\right)$, which is a contradiction. As a result, $\lambda \in \sigma\left(A_{p}\right)$. However this is absurd because $\sigma\left(A_{p}\right) \cap \mathscr{Z}\left(\Sigma_{p}\right)=\varnothing$. Therefore, $\lambda \in \mathscr{E}\left(\Sigma_{p}\right)$.

Lemma 20. If $\lambda \in \sigma(A)$ is unobservable then $E_{\lambda}(A) \subseteq \mathcal{N}(\bar{C})$.

Proof. From Lemma $19 \lambda \in \mathscr{Z}\left(\Sigma_{p}\right) \cap \sigma\left(A_{r}\right)$. The minimal realization of $\Sigma_{p}$ implies $\lambda \notin \sigma\left(A_{p}\right)$. Consider $v \in E_{\lambda}(A)$. The invertibility of $\left(A_{p}-\lambda I\right)$ guarantees that $v_{p}=-\left(A_{p}-\right.$ $\lambda I)^{-1} B_{p} C_{r} v_{r}$. On the other hand $C_{p} v_{p}=0$ since $\lambda \in \mathscr{Z}\left(\Sigma_{p}\right)$. Henceforth $v \in \mathcal{N}(\bar{C})$.

From Lemma 20 and the PBH test it follows that $E_{\lambda}(A) \subseteq$ $\mathcal{N}(\mathcal{O})$ with $\lambda \in \Lambda_{\bar{o}}$. Additionally $v \in E_{\lambda}(A) \cap \mathcal{N}(\mathcal{O})$ implies $v_{r} \in E_{\lambda}\left(A_{r}\right)$. If $\Sigma_{r}$ has a minimal realization, $\operatorname{dim} E_{\lambda}\left(A_{r}\right)=1$ (i.e., $A_{r}$ has only one Jordan block associated with $\lambda$ ). Given that $\lambda \in \mathscr{E}\left\{\Sigma_{p}\right\}$ and $\lambda \notin \sigma\left(A_{p}\right)$ it follows that $\left(A_{p}-\lambda I\right)$ is invertible, and then $v_{p}$ is uniquely determined by $v_{r}$; that is, $v_{p}=-\left(A_{p}-\lambda I\right)^{-1} B_{p} C_{r} v_{r}$. As a result $v_{p} \in \operatorname{span}\left(\left(A_{p}-\right.\right.$ $\left.\lambda I)^{-1} B_{p} C_{r} v_{r}\right)$ and

$$
v \in \operatorname{span}\left(\left(\begin{array}{c}
\left(A_{p}-\lambda I\right)^{-1} B_{p} C_{r} \\
I
\end{array}\right) v_{r}\right)=E_{\lambda}(A) .
$$

This means that $\operatorname{dim} E_{\lambda}(A)=1\left(m_{\lambda}(A)=1\right)$ and that we only need to account for one of the eigenvectors of $E_{\lambda}(A)$ to apply the PBH test. Given that we have only one Jordan block $J_{\lambda}(A)$ associated with $\lambda \in \sigma(A)$, there exists only one $A$-cyclic subspace $Z_{\lambda}(u, A)$.

Now we need the following technical lemma around the multiple zeros of a SISO system.

Lemma 21. A necessary and sufficient condition for $z \in \mathbb{C}$ to be a zero of multiplicity $m$ of the SISO system $\Sigma=(A, B, C)$ (with transfer function $\left.G(s)=Y(s) / U(s)=C(s I-A)^{-1} B\right)$ is

$$
\begin{aligned}
& C(A-z I)^{-k} B=0 \\
& \quad \text { for } k=1, \ldots, m, 0 \neq C(A-z I)^{-(m+1)} B<\infty .
\end{aligned}
$$

Proof. For more details the reader is referred to Appendices.

In the following proposition we state sufficient conditions for $Z_{\lambda}(v, A)$ to be in $\mathcal{N}(\mathcal{O})$ in terms of the existence of a multiple zero in the plant.

Proposition 22. A sufficient condition for $Z_{\lambda}(v, A)$ to be in $\mathscr{N}(\mathcal{O})$ is that $\lambda$ should be a zero of $\Sigma_{p}$ with multiplicity $m=$ $\operatorname{dim} Z_{\lambda}(v, A)$.

Proof. Assume that $\lambda \in \mathscr{Z}\left(\Sigma_{p}\right)$ with multiplicity $m$. We proceed recurrently through the tower of generalized eigenvectors from top to bottom by connecting $v^{(k)}$ to $v^{(k+1)}$ through the following problem:

$$
\left(\begin{array}{c}
v_{p}^{(k+1)} \\
v_{r}^{(k+1)}
\end{array}\right)=\left(\begin{array}{cc}
A_{p}-\lambda I & B_{p} C_{r} \\
-B_{r} C_{p} & A_{r}-\lambda I
\end{array}\right)\left(\begin{array}{c}
v_{p}^{(k)} \\
v_{r}^{(k)}
\end{array}\right) .
$$

As a result we have that $v_{p}^{(k)}$ is a function of $v_{r}^{(k)}, v_{r}^{(k+1)}, \ldots$, $v_{r}^{(m)}$ :

$$
\begin{gathered}
v_{p}^{(m)}=-\left(A_{p}-\lambda I\right)^{-1} B_{p} C_{r} v_{r}^{(m)}, \\
v_{p}^{(m-1)}=\sum_{j=1}^{2}(-1)^{j}\left(A_{p}-\lambda I\right)^{-j} B_{p} C_{r} v_{r}^{(m+j-2)}, \\
v_{p}^{(m-2)}=\sum_{j=1}^{3}(-1)^{j}\left(A_{p}-\lambda I\right)^{-j} B_{p} C_{r} v_{r}^{(m+j-3)}, \\
\vdots \\
v_{p}^{(k)}=\sum_{j=1}^{m-k+1}(-1)^{j}\left(A_{p}-\lambda I\right)^{-j} B_{p} C_{r} v_{r}^{(j+k-1)}, \\
\vdots \\
v=v_{p}^{(1)}=\sum_{j=1}^{m}(-1)^{j}\left(A_{p}-\lambda I\right)^{-j} B_{p} C_{r} v_{r}^{(j)} .
\end{gathered}
$$


In virtue of Lemma $21, C_{p}\left(A_{p}-\lambda I\right)^{-k} B_{p}=0$ for $k=$ $1,2, \ldots, m$, so $C_{p} v_{p}^{(k)}=\bar{C} v^{(k)}=0$. Henceforth $Z_{\lambda}(v, A) \subseteq$ $\mathcal{N}(\bar{C})$. On the other hand $Z_{\lambda}(v, A)$ is $A$-invariant and $v^{(1)}=v$; as a consequence $A^{j} v^{(1)} \in \operatorname{span}\left(v^{(1)}, \ldots, v^{(m)}\right)$ and $\bar{C} A^{j} v^{(1)}=$ 0 for $j=0,1, \ldots$, which means $v^{(1)} \in \mathcal{N}(\mathcal{O})$. From Lemma $2 Z_{\lambda}(v, A) \subseteq \mathcal{N}(\mathcal{O})$.

Because systems $\Sigma_{p}$ and $\Sigma_{r}$ are minimal it follows that they are controllable and observable. From the PBH test it is known that if $\lambda \in \sigma(A)$ is an unobservable mode with eigenvector $\left(v_{p}^{T}, v_{r}^{T}\right)^{T}$ then $C_{p} v_{p}=0$ and $\lambda \in \sigma\left(A_{r}\right)$. Now, we explore whether or not $\lambda \in \sigma\left(A_{p}\right)$ : To this purpose it is worth resorting to the external description of the feedback system by means of the closed-loop transfer function. We write the transfer functions of the controller and the plant as $G_{r}(s)=N_{r}(s) / D_{r}(s)$ and $G_{p}(s)=N_{p}(s) / D_{p}(s)$, respectively (with the numerator and the denominator being coprimes), and the closed-loop transfer function from $r$ to $y$ as

$$
\begin{aligned}
H(s) & =\frac{G_{r}(s) G_{p}(s)}{1+G_{r}(s) G_{p}(s)} \\
& =\frac{N_{r}(s) N_{p}(s)}{N_{r}(s) N_{p}(s)+D_{r}(s) D_{p}(s)} .
\end{aligned}
$$

If $\lambda \in \sigma\left(A_{p}\right)$ then $(s-\lambda) \mid D_{p}(s)$, and as a result $(s-\lambda) \mid N_{r}(s)$ (due to $\lambda \in \sigma(A)$ ). However, we have shown above that $\lambda \in \sigma\left(A_{r}\right)$ which is in contradiction with the assumption of minimality of the transfer function $G_{r}(s)$. Therefore, $\lambda \notin$ $\sigma\left(A_{p}\right)$.

If $\lambda \in \sigma(A)$ it is clear that $\lambda$ is a root of the characteristic polynomial $p_{c}(s)=N_{r}(s) N_{p}(s)+D_{r}(s) D_{p}(s)$, and then $(s-\lambda) \mid N_{r}(s) N_{p}(s)$. The minimality of the transfer function $G_{r}(s)$ together with $(s-\lambda) \mid D_{r}(s)$ (or $\lambda \in \sigma\left(A_{r}\right)$ ) implies that $(s-\lambda) \mid N_{p}(s)$. As a result, the lack of observability is caused by cancellations of zeros of $\Sigma_{p}$ with poles of $\Sigma_{r}$.

Remark 23. Assume that we reduce the closed-loop transfer function as $\widetilde{H}(s)=\widetilde{N}_{r}(s) \widetilde{N}_{p}(s) /\left(\widetilde{N}_{r}(s) \widetilde{N}_{p}(s)+\widetilde{D}_{r}(s) \widetilde{D}_{p}(s)\right)$, where $(s-\lambda) \mid \widetilde{N}_{r}(s) \widetilde{N}_{p}(s)$ but $(s-\lambda)+\widetilde{D}_{r}(s)$. If $\lambda$ is a root of the denominator of $\widetilde{H}(s)$ there exists a cancellation zero-pole. Additionally, $(s-\lambda) \mid \widetilde{D}_{r}(s) \widetilde{D}_{p}(s)$ and then $(s-\lambda) \mid \widetilde{D}_{p}(s)$ which is impossible since $(s-\lambda)+D_{p}(s)$ and $\widetilde{D}_{p}(s)$ is a divisor of $D_{p}(s)$.

In some occasions $\operatorname{dim} Z_{\lambda}(v, A)$ can be greater than the algebraic multiplicity of $\lambda$ as a pole of $\Sigma_{r}$. The following example reveals this case.

Example 24. Consider a plant $\Sigma_{p}$ with an external description $H_{p}(s)=2\left((s+1)^{2} /\left(2 s^{3}+8 s^{2}+8 s+3\right)\right)$ and an observable internal description $\Sigma_{p}=\left(A_{p}, B_{p}, C_{p}\right)$ :

$$
\begin{aligned}
& \left(\Sigma_{p}\right): \\
& \left(\begin{array}{c}
\left(\begin{array}{c}
\dot{x}_{1} \\
\dot{x}_{2} \\
\dot{x}_{3}
\end{array}\right)= \\
y=\left(\begin{array}{lll}
0 & 0 & -\frac{3}{2} \\
1 & 0 & -4 \\
0 & 1 & -4
\end{array}\right)\left(\begin{array}{l}
x_{1} \\
x_{2} \\
x_{3}
\end{array}\right)+\left(\begin{array}{l}
1 \\
2 \\
x_{1} \\
x_{2}
\end{array}\right) u \\
x_{3}
\end{array}\right)
\end{aligned}
$$

And let $\Sigma_{r}$ be a regulator system with transfer function $H_{r}(s)=(1 / 2)\left((2 s+1) /(s+1)^{2}\right)$ and an observable state-space description $\Sigma_{r}=\left(A_{r}, B_{r}, C_{r}\right)$ :

$$
\left(\Sigma_{r}\right):\left\{\begin{array}{l}
\left(\begin{array}{c}
\dot{w}_{1} \\
\dot{w}_{2}
\end{array}\right)=\left(\begin{array}{ll}
0 & -1 \\
1 & -2
\end{array}\right)\left(\begin{array}{l}
w_{1} \\
w_{2}
\end{array}\right)+\left(\begin{array}{c}
\frac{1}{2} \\
1
\end{array}\right) e \\
u=\left(\begin{array}{ll}
0 & 1
\end{array}\right)\left(\begin{array}{l}
w_{1} \\
w_{2}
\end{array}\right) .
\end{array}\right.
$$

The interconnected system $\Sigma_{r}=(A, B, C)$ via feedback has the following state-space equations:

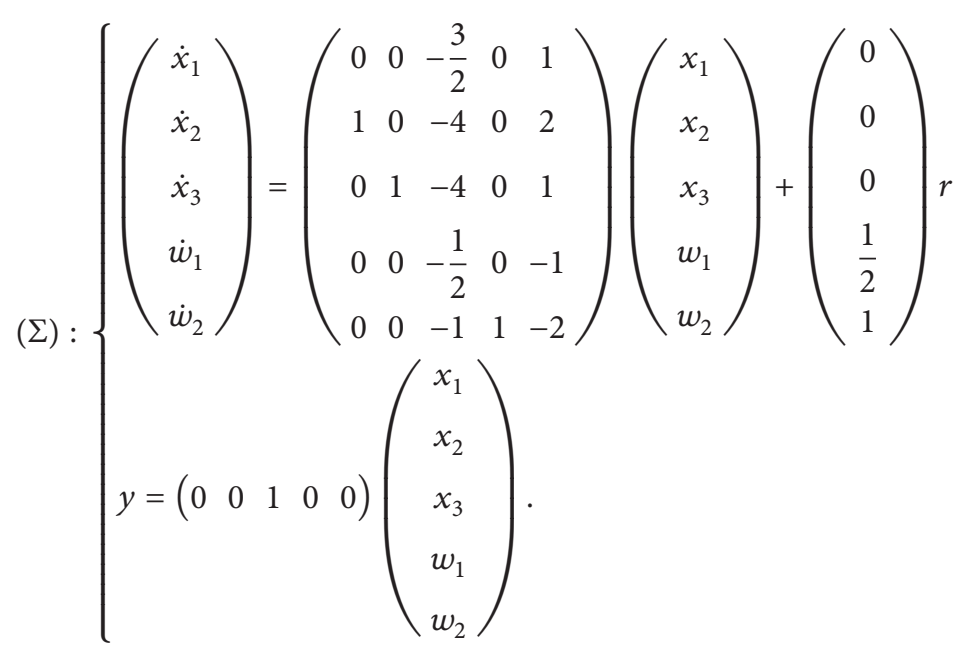


The poles of $\Sigma$ are located at $-1,-2$; that is, $\sigma(A)=\{-1,-2\}$. In virtue of the Jordan canonical decomposition we can write $\mathbb{R}^{n}=Z_{-1}(v, A) \oplus Z_{-2}(w, A)$ where $v=(0,4,2,2,1)$ and $w=$ $(0,1,2,2,3)$. According to Lemma 9

$$
\begin{aligned}
& Z_{-1}(v, A)=\operatorname{span}\left(v_{1}=v=(0,4,2,2,1), v_{2}\right. \\
& \quad=(0,2,1,2,1), v_{3}=(1,0,0,-1,0), v_{4} \\
& \quad=(1,1,0,-1,-1)), \\
& Z_{-2}(w, A)=\operatorname{span}(w),
\end{aligned}
$$

where $E_{-1}(A)=\operatorname{span}\left(v_{4}\right)$ and $E_{-2}(A)=\operatorname{span}(w)$. From the $\mathrm{PBH}$ test the mode $\lambda=-2$ is observable $(C w \neq 0)$ and $\lambda=-2$ is nonobservable $\left(C v_{4}=0\right)$. Furthermore, since $w \in E_{\lambda}(A) \cap \mathcal{N}(\mathcal{O})^{\perp}$, in virtue of Lemma 12 it follows that $Z_{\lambda}(u, A) \subseteq \mathcal{N}(\mathcal{O})^{\perp}$. However $\operatorname{span}\left(v_{3}, v_{4}\right) \subseteq \mathcal{N}(\mathcal{O})$, and $v_{1}, v_{2} \notin \mathcal{N}(\mathcal{O})$. This means that $Z_{-1}(v, A) \nsubseteq \mathcal{N}(\mathcal{O})$ and we can not derive a spectral decomposition of $\mathcal{N}(\mathcal{O})$ as a direct sum of root spaces. Note that in this case we have the following closed-loop transfer function:

$$
\begin{aligned}
& H(s) \\
& \quad=\frac{(s+1)^{2}(2 s+1)}{(s+1)^{2}(2 s+1)+(s+1)^{2}\left(2 s^{3}+8 s^{2}+8 s+3\right)} \\
& \quad=\frac{(s+1)^{2}(2 s+1)}{2(s+2)(s+1)^{4}} .
\end{aligned}
$$

In this case there exists a cancellation of two poles of the regulator with two zeros of the plant at $s=-1$, but the feedback generates two additional closed-loop poles at $s=-1$. Thus, we have a unique Jordan block $J_{-1}(A)$ of dimension 4 and an unobservable invariant subspace $\operatorname{span}\left(v_{3}, v_{4}\right)$. Henceforth, we conclude that $\mathcal{N}(\mathcal{O})=\operatorname{span}\left(v_{3}, v_{4}\right)$.

Proposition 25. A necessary condition for $Z_{\lambda}(v, A)$ to be in $\mathcal{N}(\mathcal{O})$ is $\lambda$ to be a pole of $\Sigma_{r}$ with multiplicity $\operatorname{dim} Z_{\lambda}(v, A)$.

Proof. If $Z_{\lambda}(v, A) \subseteq \mathcal{N}(\mathcal{O})$ with $\operatorname{dim} Z_{\lambda}(v, A)=m$, then $C_{p} v_{p}^{(k)}=0$ for $k=1,2, \ldots, m$. This implies that $\left(A_{r}-\right.$ $\lambda I) v_{r}^{(k-1)}=v_{r}^{(k)}$; that is, $v_{r}^{(k)}$ is a generalized eigenvector associated with $\lambda$ in $\Sigma_{r}$. Therefore $\lambda$ is a pole of $\Sigma_{r}$ with multiplicity $m$.

Example 26. We have one unobservable mode $\lambda=-1$ with three subcyclic subspaces $Z_{-1}\left(e_{5}, A\right), Z_{-1}\left(e_{6}, A\right)$, and $Z_{-1}\left(e_{7}, A\right)$ and the root subspace $\mathcal{N}_{-1}=Z_{-1}\left(e_{5}, A\right) \oplus$ $Z_{-1}\left(e_{6}, A\right) \oplus Z_{-1}\left(e_{7}, A\right)$.

From Propositions 25 and 22 we conclude that a necessary and sufficient condition for $Z_{\lambda}(v, A)$ to be in $\mathcal{N}(\mathcal{O})$ is that there should exist exactly $\operatorname{dim} Z_{\lambda}(v, A)$ cancellations between zeros of the plant and poles of the regulator. Owing to the fact that systems $\Sigma_{p}$ and $\Sigma_{r}$ are minimal it follows that they are controllable and observable. From the Popov-BelevitchHautus test ( $\mathrm{PBH}$ test) it is known that if $\lambda \in \sigma(A)$ is an unobservable mode with eigenvector $\left(v_{p}^{T}, v_{r}^{T}\right)^{T}$ then $C_{p} v_{p}=0$ and $\lambda \in \sigma\left(A_{r}\right)$. Furthermore, the single output system $\Sigma_{r}$ is observable if and only if in the Jordan form matrix there is one Jordan block associated with each distinct eigenvalue and every entry of $C_{r}$ corresponding to the last column of each Jordan block is nonzero. For an unobservable mode $\lambda \in \sigma(A)$, if $m_{\lambda}(A)=1$ we have that $\mathcal{N}_{\lambda}$ is $A$-cyclic and as a consequence we can determine whether or not $\mathcal{N}_{\lambda} \subseteq \mathcal{N}(\mathcal{O})$ in terms of poles and zeros.

\section{Discussion}

The canonical structure of the unobservable subspace as a direct sum of cyclic subspaces (spectral decomposition) has been analyzed via Kalman's decomposition. Necessary and sufficient conditions have been provided for the unobservable subspace to be spectral for the system matrix $A$; specifically, when a cyclic subspace has an eigenvector in the orthogonal complement of the unobservable subspace then it is strictly included in this subspace. This property does not always hold for the unobservable subspace, but rather we can state at most that the unobservable cyclic subspaces are included in a cyclic subspace from the Jordan decomposition. This analysis leads to determining the conditions of $A$-invariance for the intersection of the unobservable subspace with an arbitrary subspace. Finally, spectral decomposition of the unobservable subspace is connected to the existence of multiple transmission zeros in feedback systems.

\section{Appendices}

\section{A. Multiple Transmission Zeros}

This section is devoted to present the proof of technical Lemma 21.

Proof. Consider the following.

Sufficiency. From the definition of SISO system $\Sigma$ in the statespace form the following identities are in order:

$$
\begin{aligned}
X(s) & =(s I-A)^{-1} B U(s), \\
(s-z) X(s) & =(A-z I) X(s)+B U(s) .
\end{aligned}
$$

Finding $X(s)$ from (A.2) yields

$$
X(s)=(A-z I)^{-1}[(s-z) X(s)-B U(s)]
$$

and by definition of zero of the system $\Sigma$,

$$
G(z)=-C(A-z I)^{-1} B=0 .
$$

Thus,

$$
Y(s)=C X(s)=(s-z) C(A-z I)^{-1} X(s) .
$$

Now folding (A.2) into the last expression leads to

$$
Y(s)=-(s-z) C(A-z I)^{-1}(A-s I)^{-1} B U(s)
$$


and as a consequence,

$$
\frac{G(s)}{s-z}=-C(A-z I)^{-1}(A-s I)^{-1} B
$$

On the other hand we have assumed that $z$ is a multiple zero so the following identity is immediate:

$$
\left.\frac{G(s)}{s-z}\right|_{s=z}=-C(A-z I)^{-2} B=0 .
$$

In a similar way,

$$
\begin{aligned}
(s-z)^{2} X(s)= & (A-z I)^{2} X(s) \\
& +[(A-z I)+(s-z) I] B U(s)
\end{aligned}
$$

and finding $X(s)$ on the right hand of this equation we obtain

$$
\begin{aligned}
X(s)= & (A-z I)^{-2}(s-z)^{2} X(s) \\
& -(A-z I)^{-1} B U(s) \\
& -(s-z)(A-z I)^{-2} B U(s) .
\end{aligned}
$$

Using (A.8) and (A.4), the output is simplified into

$$
\begin{aligned}
Y(s) & =C X(s) \\
& =(s-z)^{2} C(A-z I)^{-2}(s I-A)^{-1} B U(s) ;
\end{aligned}
$$

that is,

$$
\frac{G(s)}{(s-z)^{2}}=-C(A-z I)^{-2}(A-s I)^{-1} B
$$

In virtue of the multiplicity of the zero $s=z$ we conclude that

$$
\left.\frac{G(s)}{(s-z)^{2}}\right|_{s=z}=-C(A-z I)^{-3} B=0
$$

and repeating successively this procedure results in

$$
\begin{aligned}
&\left.\frac{G(s)}{(s-z)^{k}}\right|_{s=z}=-C(A-z I)^{-(k+1)} B=0 \\
& \text { for } k=1, \ldots,(m-1) .
\end{aligned}
$$

Necessity. The proof is straightforward. If $C(A-z I)^{-k} B=0$ for $k=1, \ldots, m$ then $\left.\left(G(s) /(s-z)^{(k-1)}\right)\right|_{s=z}=-C(A-z I)^{-k} B=0$ for $k=1, \ldots, m$, and additionally $\left.\left(G(s) /(s-z)^{m}\right)\right|_{s=z}=$ $-C(A-z I)^{-(m+1)} B \neq 0$, so that $z$ is a zero of the system $\Sigma$.

Remark A.1. An alternative proof of this is based on the derivative of the inverse function of a linear transformation with matrix $M(s) \in \mathbb{C}^{n \times n}$ (this is a simple application of the chain rule):

$$
\begin{aligned}
\frac{d}{d s}\left[M^{-1}(s) M(s)\right]= & M^{-1}(s) \frac{d M(s)}{d s} \\
& +\frac{d M^{-1}(s)}{d s} M(s)=0 .
\end{aligned}
$$

From this it follows that

$$
\frac{d M^{-1}(s)}{d s}=-M^{-1}(s) \frac{d M(s)}{d s} M^{-1}(s) .
$$

Let $H(s)=C(s I-A)^{-1} B$ be the transfer function of the system $\Sigma$ and $z \in \mathbb{C}$ a zero of $H$. Applying the last identity with $M(s)=(s I-A)$ we derive the following result:

$$
\frac{d H(s)}{d s}=C \frac{d}{d s}(s I-A)^{-1} B=-C(s I-A)^{-2} B .
$$

Now if we apply the same procedure to $M(s)=(s I-A)^{2}$ we obtain

$$
\begin{aligned}
\frac{d^{2} H(s)}{d s^{2}} & =-C \frac{d}{d s}\left[(s I-A)^{2}\right]^{-1} B \\
& =2 C(s I-A)^{-2}(s I-A)(s I-A)^{-2} B \\
& =2 C(s I-A)^{-3} B
\end{aligned}
$$

and so it is easy to check the identity

$$
\frac{d^{k} H(s)}{d s^{k}}=(-1)^{k} k ! C(s I-A)^{-(k+1)} B .
$$

For $z$ to be a zero of multiplicity $m$ of $H$, it is necessary that $\left.\left(d^{k} H(s) / d s^{k}\right)\right|_{s=z}=0$ for $k=0,1, \ldots,(m-1)$. According to (A.19) this is equivalent to

$$
C(z I-A)^{-k} B=0 \quad \text { para } k=1, \ldots, m
$$

which is the same expression that we delivered in the technical lemma.

\section{B. Nonminimal Transfer Functions}

In this section we illustrate how a nonminimal transfer function (reducible rational function) can be represented in the space-state form.

Example B.1. Let $\Sigma$ be an unobservable system with nonminimal transfer function $H(s)=Y(s) / U(s)=(s+1)^{2} /(s+1)^{4}$. Its expansion in partial fractions exhibits two zero residues:

$$
\begin{aligned}
H & (s) \\
& =\lim _{\epsilon \rightarrow 0}\left(\frac{\epsilon}{(s+1)^{4}}+\frac{\epsilon}{(s+1)^{3}}+\frac{C_{2}}{(s+1)^{2}}+\frac{C_{1}}{(s+1)}\right) .
\end{aligned}
$$

We define the states $X_{k}(s)=\left(1 /(s+1)^{4-k+1}\right) U(s)$ for $k=1,2$, 3,4 which leads to the relations $X_{1}(s) / X_{2}(s)=X_{2}(s) / X_{3}(s)=$ $X_{3}(s) / X_{4}(s)=1 /(s+1)$. As a result, we can express $\Sigma$ in the Jordan form: 


$$
(\Sigma):\left\{\begin{array}{c}
\left(\begin{array}{c}
\dot{x}_{1} \\
\dot{x}_{2} \\
\dot{x}_{3} \\
\dot{x}_{4}
\end{array}\right)=\left(\begin{array}{cccc}
-1 & 1 & 0 & 0 \\
0 & -1 & 1 & 0 \\
0 & 0 & -1 & 1 \\
0 & 0 & 0 & -1
\end{array}\right) \\
y=\left(\begin{array}{llll}
\epsilon & \epsilon & C_{2} & C_{1}
\end{array}\right)\left(\begin{array}{l}
x_{1} \\
x_{1} \\
x_{2} \\
x_{3} \\
x_{3} \\
x_{4}
\end{array}\right)+
\end{array}\right.
$$

As $\epsilon \rightarrow 0$ the vector of observations tends to $\left(\begin{array}{llll}0 & 0 & C_{2} & C_{1}\end{array}\right)$; that is, there exist states that do not contribute to the output, as expected from the unobservability of $\Sigma$.

\section{Conflict of Interests}

The authors declare that there is no conflict of interests regarding the publication of this paper.

\section{References}

[1] E. Hendricks, O. Jannerup, and P. Sørensen, Linear Systems Control: Deterministic and Stochastic Methods, Springer, Berlin, Germany, 2008.

[2] K. Zhou, J. C. Doyle, and K. Glover, Robust and Optimal Control, Prentice Hall, Upper Saddle River, NJ, USA, 1996.

[3] H. L. Trentelman, A. A. Stoorvogel, and M. Hautus, Control Theory for Linear Systems, Communications and Control Engineering, Springer, 2001.

[4] G. Basile and G. Marro, "Controlled and conditioned invariant subspaces in linear system theory," Journal of Optimization Theory and Applications, vol. 3, no. 5, pp. 306-315, 1969.

[5] A. Baños and J. I. Mulero, "Well-posedness of reset control systems as state-dependent impulsive dynamical systems," Abstract and Applied Analysis, vol. 2012, Article ID 808290, 16 pages, 2012.

[6] P. Antsaklis and A. Michel, A Linear Systems Primer, Birkhäuser, Boston, Mass, USA, 2007.

[7] I. Gohberg, P. Lancaster, and L. Rodman, Invariant Subspaces of Matrices with Applications, Classics in Applied Mathematics (Book 51), Society for Industrial and Applied Mathematics (SIAM), Philadelphia, Pa, USA, 2006.

[8] F. Gantmacher, The Theory of Matrices I, Chelsea Publishing, AMS Chelsea Publishing, 1959.

[9] D. H. Owens, "Invariant zeros of multivariable systems: a geometric analysis," International Journal of Control, vol. 26, no. 4, pp. 537-548, 1977. 


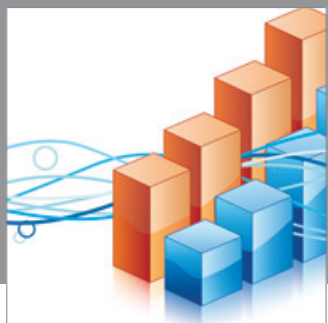

Advances in

Operations Research

mansans

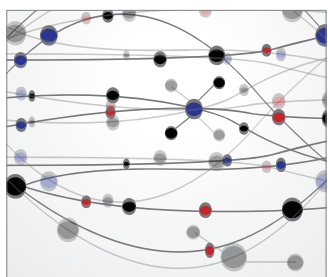

The Scientific World Journal
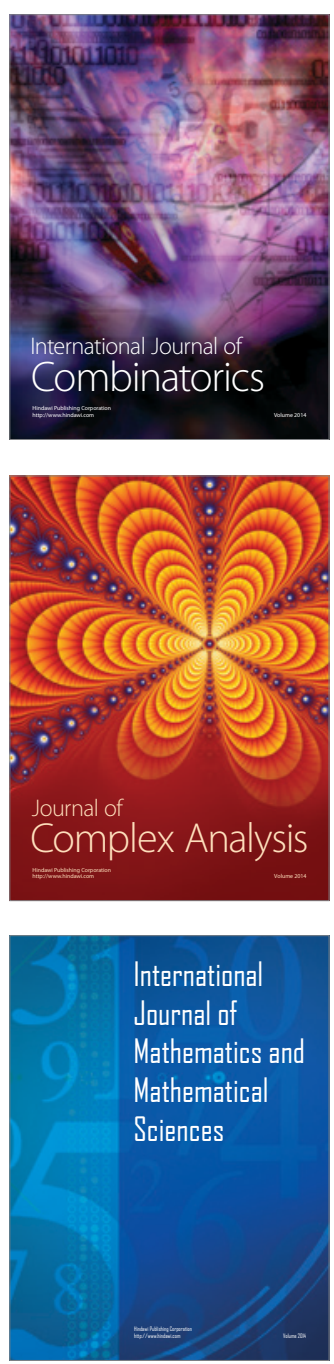
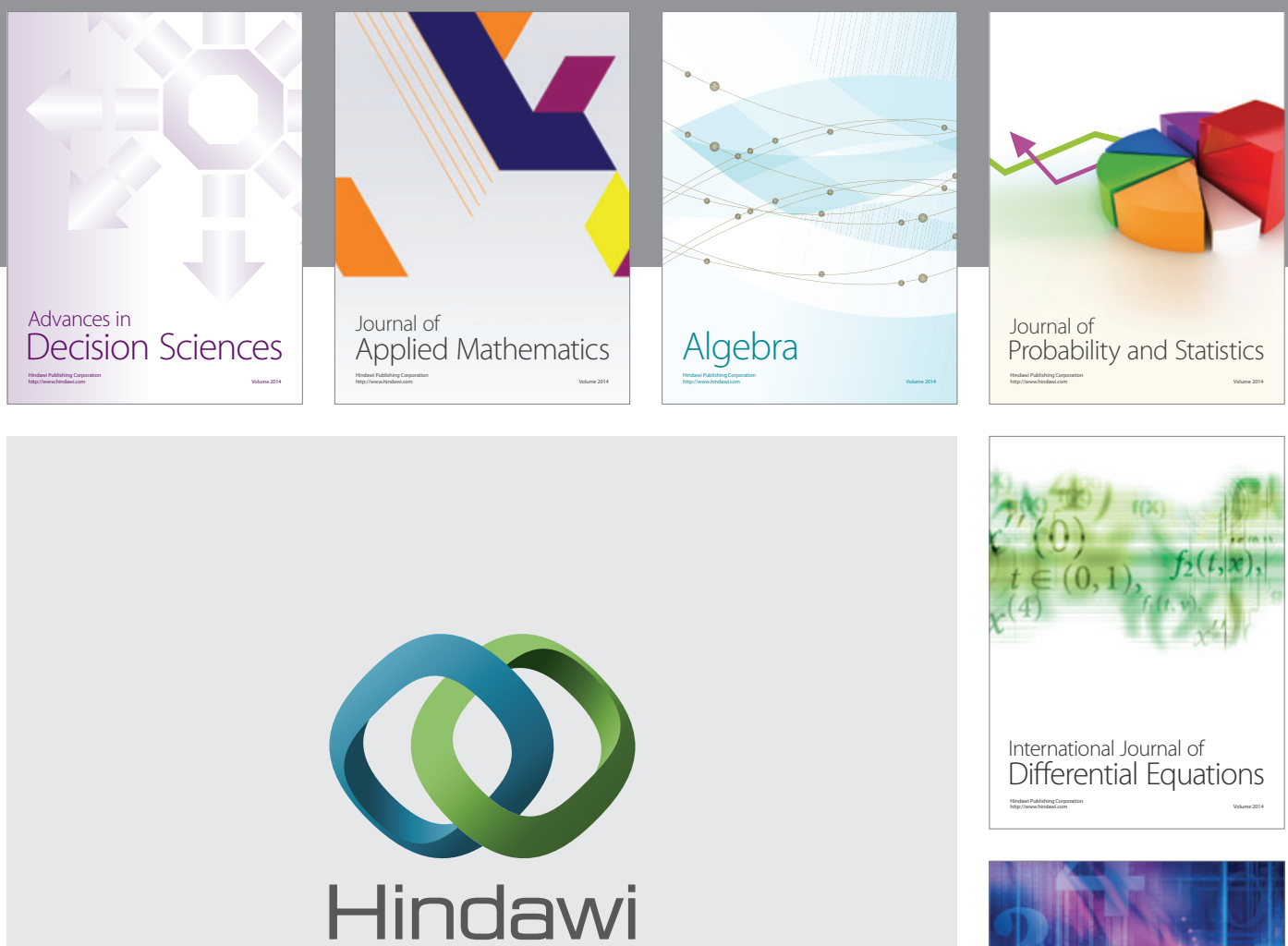

Submit your manuscripts at http://www.hindawi.com
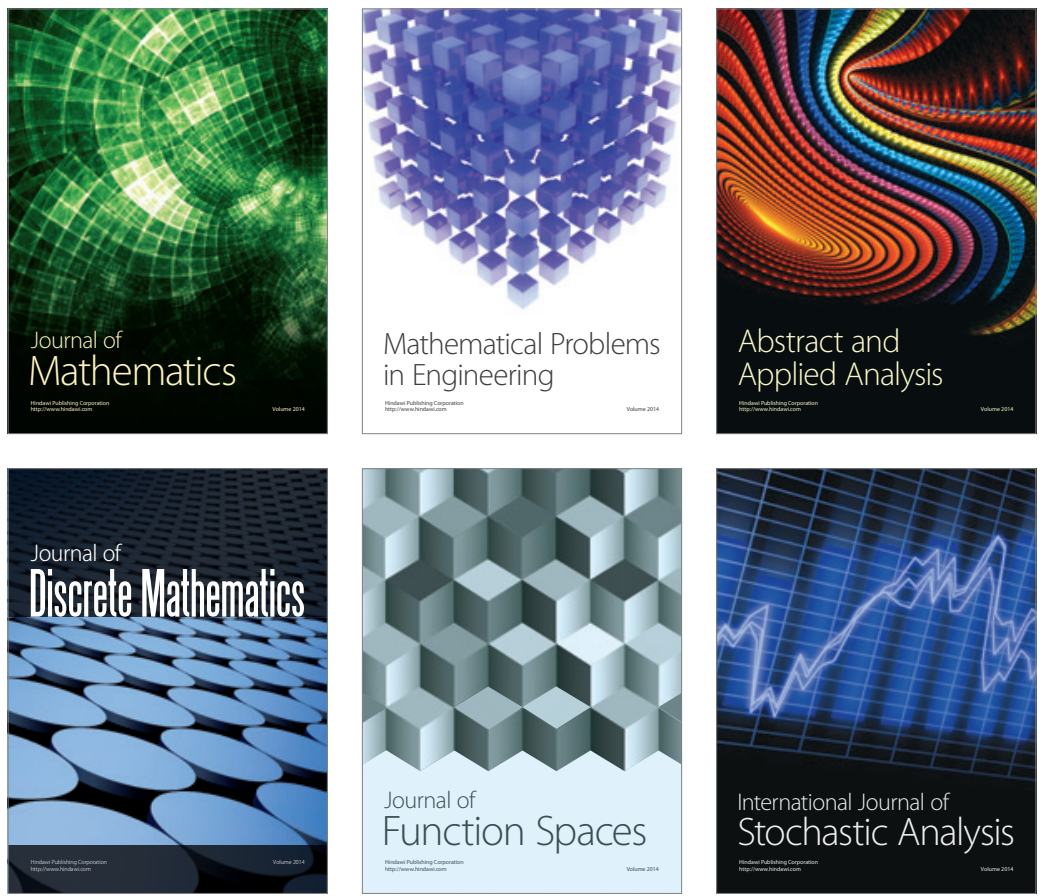

Journal of

Function Spaces

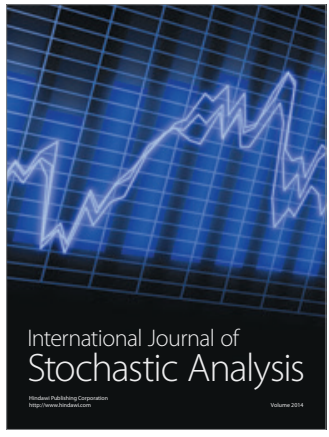

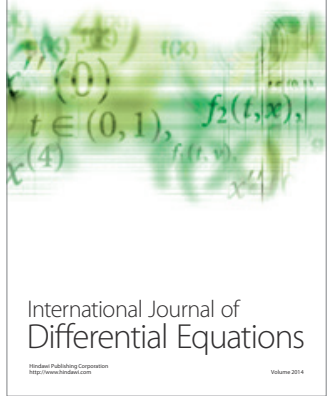
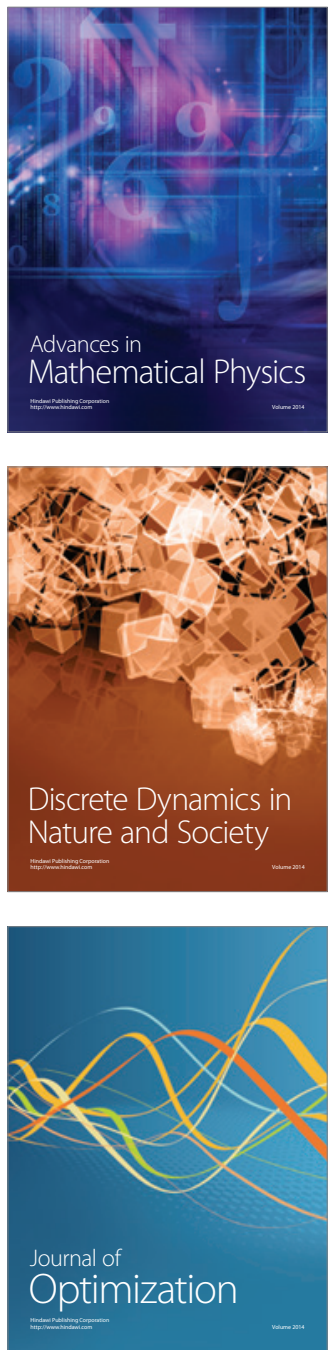